\title{
Numerical Investigation into the Air Flow Distributions of the Air Conditioning System in the Modular Data Center
}

\author{
Hao Dong ${ }^{1}$, Ziqiang Qin ${ }^{2}$, Shicheng $\mathrm{Liu}^{1}$, Yao $\mathrm{Li}^{2}$, Yingying Shen ${ }^{2}$, \\ Haibo Wang ${ }^{2}$, Yitong Zong ${ }^{2}$, Xiaojun $\mathrm{Wu}^{3}$ and Haiqing $\mathrm{Si}^{2, *}$ \\ ${ }^{1}$ College of Aerospace Engineering, Nanjing University of Aeronautics and \\ Astronautics, Nanjing 210016, China \\ ${ }^{2}$ College of Civil Aviation and Flight, Nanjing University of Aeronautics and \\ Astronautics, Nanjing 210016, China \\ ${ }^{3}$ China Aerodynamics Research and Development Center, Mianyang 621000, China
}

Received 6 June 2018, Accepted (in revised version) 4 July 2018

\begin{abstract}
Data center plays an increasingly important role in everyday life. As data center is becoming more and more powerful, energy consumption is also increasing dramatically. The air conditioning system occupies at least 50 percent of the total energy consumption. Therefore, delicate analysis on the air conditioning system could help to reduce energy consumption in data center. An advanced Finite Volume Method with RNG $k-\varepsilon$ model and convective heat exchange model is used in this paper to study the airflow and the temperature distribution of modular data center under different arrangements. Specifically, the calculation formula of convective heat transfer coefficient for plate flow is adopted to simplify analysis; and fans on the back of racks are simplified to be walls with a certain pressure jump. Simulations reveal that, in the case where air conditioners are arranged face-to-face, the temperature distribution on the back of racks is not uniform, and local high temperature points emerge near the side wall of air conditioners. By analyzing the distribution of air flow and temperature, geometric model is optimized by using a diagonal rack arrangement and drilling holes on the side wall. In the same energy consumption situation, the overall maximum temperature of the optimized model is reduced by $2.3^{\circ} \mathrm{C}$ compared with that of the original one, and the maximum temperature on the server surface is reduced by $1^{\circ} \mathrm{C}$. Based on the optimized model, the effect of the hot aisle distance on the temperature distribution is studied. By simulating four different cases with various distances of hot aisle of $100 \mathrm{~cm}, 120 \mathrm{~cm}, 130 \mathrm{~cm}$ and $150 \mathrm{~cm}$, it is found that the temperature is generally lower and distributed more evenly in the case with $120 \mathrm{~cm}$ hot aisle distance. This demonstrates that the distance of hot aisle has an effect on temperature.
\end{abstract}

AMS subject classifications: 58D30, 76N15, 76N25, 76B47

Key words: Modular data center, air conditioning, temperature distribution, hot/cold aisle, CFD.

*Corresponding author.

Email: sihaiqing@126.com (H. Q.Si) 


\section{Introduction}

Data center is the physical space for centralized processing, storage, transmission, exchange, and management of information. Continuous developments of micro-electronic technology and chip integration enable significant improvements in packaging density and operating frequency, which is also accompanied by rapid increase in the heat flux density. In cloud computing data center, power consumption can reach $10 \mathrm{~kW}$ for a single rack. The reliability and service time of a server largely depend on its operating temperature. Overheating of the device can seriously affect its stability and reliability [1]. Due to that, effective and efficient air conditioning system is an essential part of the data center. Scientific organization of air flow in the air conditioning system not only fulfills temperature requirements of data center [2], but also reduces energy consumption. Data center is one of the largest energy-consuming facilities mainly in many developed countries. Traditional temperature control solution accounts for 30\% to 50\% [3-5] of the total power consumption of the data center. Therefore, exploration in reducing PUE value (a ratio of air conditioning system energy consumption to the total energy consumption) and creating an energy-saving and environmentally friendly data center would be a very interesting research topic academically and economically.

The servers used in high-density data centers are generally blade severs, the size and power consumption of each sever are fixed. The cold air supplying from the airconditioning flows over the sever surface which brings away heat through the convection effect. Therefore, studying and understanding the distribution of airflow could favor improving the cooling efficiency of air conditioning. Yang et al. simulated the temperature field of air-cooled radiator [6]. According to the theory of thermal convection [7], a physical model was established and formulas of calculating the convective heat transfer coefficient were given. Through comparing with the results of simulation and experiments, the correctness of the calculation method for convective heat transfer coefficient was verified. Since the heat dissipation process of blade sever is similar to that of the aircooled radiator, the same formula will be utilized in this work to quantify the convective heat transfer coefficient.

The widespread use of data centers and the drastic increase of power consumption have made the thermal management of data center more difficult. Most computer servers are air-cooled in typical data centers. Cho and Kim [8] pointed that coolant air mixing with heated air exhausted from equipments would lower the efficiency of the air conditioning system. Vaibhav et al. [9] carried out some experiments about the inlet temperature of racks in both open and contained aisle conditions. Hot air will mix with cold air in open cold aisle. Even if over-providing cold air, hot air still exists on the top of cold aisle. Nada et al. [10] investigated, compared and evaluated three different thermal management solutions of data center. In their experiments, the hot aisle and the cold aisle are in different sides of the rack. They found that the rack inlet air temperature was reduced by $3-13 \%$ for aisle partition configuration, while the reduction reaches $13-15.5 \%$ for aisle containment configuration. Apart from establishing the hot and the cold aisles, many 
other methods have been proposed to further increase the cooling efficiency. Priyadumkol and Kittichaikarn [11] studied three different systems, including the raised floor and the in-row air conditioning systems, and the combinations with them. Their simulation results agreed well with the measured data. Specifically, the in-row air conditioning system performs better than the raised floor air conditioning system in delivering cold air to the top of racks. For lower racks, the raised floor air conditioning system is more efficiency. Minimizing hot air re-circulation and reducing cold air loss are two essential factors in enhancing thermal conditions and saving energy. Studies was carried out and revealed the optimal temperatures for the aisle partition and containment configurations as $18^{\circ} \mathrm{C}$ and $22^{\circ} \mathrm{C}$, respectively [12]. Though the aisle containment configuration has many advantages, there are considerations that must be paid attention to, for example, unit airflow must closely match with the cumulative server airflow [13]. In under-floor data centers, cold aisle containment can reduce the exhaust air temperature of racks and local hotspots, but air temperature distribution of racks is still uneven. Baffles which are under floor can not only further reduce the temperature, but also make it more uniform. Yuan et al. found that when the angle between baffles and computer room air conditioners outlet air orientation is at 45 degrees, temperature of local hotspots drops from $30.5^{\circ} \mathrm{C}$ to $28.4^{\circ} \mathrm{C}$ and air distribution is the most even [14].

Compared with traditional configuration of data center, modular air conditioning system is becoming popular due to its advantages of quick construction, easy replication, rapid deployment, flexible scalability, and high cooling efficiency [15]. Two major contributors to improving cooling efficiency of the modular air conditioning system are still reducing mixing of cold air and hot air and improving the uniformity of airflow distribution. Therefore, conventional methods of increasing cooling efficiency can also be used in the modular data center. Closed cold aisle is used in this paper because it has been verified by many researchers $[3,10,22]$ and is a good way to avoid mixing hot air with cold air. The uniformity of airflow distribution directly affects the temperature. Ideas of making the air flow more uniform by adjusting configurations is worth adopting. In the simulation results of modular data center, it was found that temperature of servers would be higher near both sides of the air conditioner and both sides of the cabinet, which is due to the wall effect that hinders the air flow from bringing heats out. Therefore, changing physical structure of cabinets can possibly make the air distribution more uniform. In heat transfer process, the width of hot aisle also affects the efficiency of air conditioning system. By adjusting the distance of the hot aisle, the optimal location of the hot aisle can be found. The above two points were usually ignored or not discussed in detail in previous studies. Efforts will be made in this paper to find the answers.

\section{Geometric model}

A simplified modular air conditioning system room with $6.6 \mathrm{~m} \times 5.8 \mathrm{~m} \times 3.3 \mathrm{~m}$ (length $\times$ width $\times$ height) is set up and shown in Fig. 1. In every column, there are 


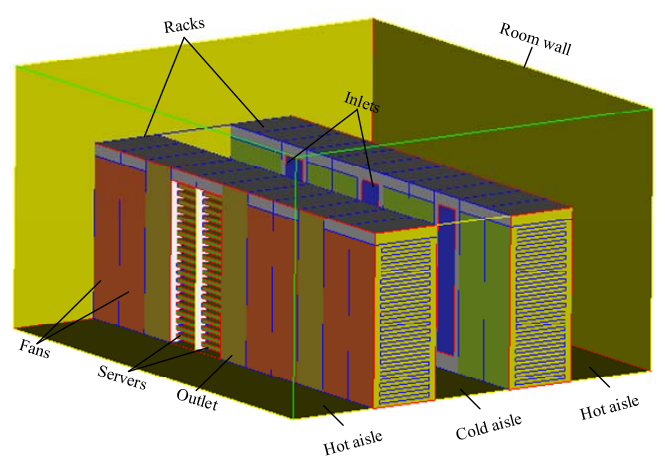

Figure 1: Model of modular data center.

8 racks and 3 air conditioners, half of the quantity of Liu's model for hardware limitations [15]. Cold aisle between two columns is containment for cold air. Hot aisle is in the back of racks. Cold air coming from inlets goes through the racks, servers with power of $10 \mathrm{kw}$, fans and returns back to outlets of air conditioners. Each rack contains 20 severs with the interval distance $50 \mathrm{~mm}$ between each other, and several fans which are simplified as a wall in the back. Other details are in the Table 1.

Table 1: Sizes for model.

\begin{tabular}{||l|c||}
\hline Items & Size \\
\hline Room & $6.6 \mathrm{~m} \times 5.8 \mathrm{~m} \times 3.3 \mathrm{~m}$ \\
Cold aisle & $6.6 \mathrm{~m} \times 1.2 \mathrm{~m} \times 2.2 \mathrm{~m}$ \\
Hot aisle & $6.6 \mathrm{~m} \times 1.3 \mathrm{~m} \times 2.2$ \\
Rack & $600 \mathrm{~mm} \times 1000 \mathrm{~m} \times 2200 \mathrm{~mm}$ \\
Sever & $800 \mathrm{~mm} \times 600 \mathrm{~mm} \times 50 \mathrm{~mm}$ \\
Inlet & $400 \mathrm{~mm} \times 1850 \mathrm{~mm}$ \\
Outlet & $600 \mathrm{~mm} \times 1000 \mathrm{~mm} \times 2200 \mathrm{~mm}$ \\
Fan & $600 \mathrm{~mm} \times 2050 \mathrm{~mm}$ \\
\hline
\end{tabular}

\section{Numerical study}

After setting up geometric structures of air conditioning system, numerical simulation models should be established to solve real problems. To validate the present model, it is common to compare numerical simulation results with experimental data.

\subsection{Numerical model}

ANSYS CFD 15.0 is used to solve this steady, 3D and turbulent problem. It is important to choose a correct viscous model. According to physical conditions, its Reynolds numbers 
reach to $1.5 \times 10^{5}$ at least. There are many optional turbulence models developed for RANS model, such as standard $k-\varepsilon$ model, RNG $k-\varepsilon$ model, Spalar-Allmaras model, and so on. In this case, we choose RNG $k-\varepsilon$ model, which is the most suitable vicious model for indoor air conditioning system [16]. Mass continuity equation, momentum equation, energy equation, RNG $k-\varepsilon$ model and buoyancy model are applied in the simulation. The conservation equations and transport equations are listed below:

$$
\begin{aligned}
& \frac{\partial \rho}{\partial \mathrm{t}}+\nabla \cdot(\rho \vec{v})=S_{m}, \\
& \frac{\partial(\rho \vec{v})}{\partial \mathrm{t}}+\nabla \cdot(\rho \vec{v} \vec{v})=-\nabla p+\nabla(\bar{\tau})+\rho \vec{g}+\vec{F} \\
& \frac{\partial}{\partial \mathrm{t}}\left(\rho\left(h-\frac{p}{\rho}+\frac{v^{2}}{2}\right)\right)+\nabla \cdot\left(\vec{v}\left(\rho\left(h-\frac{p}{\rho}+\frac{v^{2}}{2}\right)+p\right)\right) \\
& =\nabla \cdot\left(k_{e f f} \nabla T-\sum_{j} h_{j} \vec{J}_{j}+\left(\bar{\tau}_{e f f} \cdot \vec{v}\right)\right)+S_{h \prime} \\
& \frac{\partial(\rho k)}{\partial \mathrm{t}}+\frac{\partial}{\partial x_{i}} \cdot\left(\rho k u_{i}\right)=\frac{\partial}{\partial x_{j}} \cdot\left(\alpha_{k} \mu_{e f f} \frac{\partial k}{\partial x_{j}}\right)+G_{k}+G_{b}-\rho \varepsilon-Y_{M}+S_{k \prime} \\
& \frac{\partial(\rho \varepsilon)}{\partial t}+\frac{\partial}{\partial x_{i}} \cdot\left(\rho \varepsilon u_{i}\right)=\frac{\partial}{\partial x_{j}} \cdot\left(\alpha_{k} u_{e f f} \frac{\partial \varepsilon}{\partial x_{j}}\right)+C_{1 \varepsilon} \frac{\varepsilon}{k}\left(G_{k}+C_{3 \varepsilon} G_{b}\right)-C_{2 \varepsilon} \rho \frac{\varepsilon^{2}}{k}-R \varepsilon+S_{\varepsilon} .
\end{aligned}
$$

The finite-volume method is used to solve the conservation equations. The momentum and energy equations are discretized using second-order upwind scheme, and the other equations are discretized by first-order upwind scheme. In this simulation air is incompressible and follows the Boussinesq hypothesis that changes in density only affect the buoyancy force term and have no effect on other items. Non-slip condition is applied in all the walls in the calculation, and standard wall functions are used to treat near walls. The residual value of energy is set to be $10^{-6}$, other residual values are set to be $10^{-3}$ [17].

The heat dissipation of electronic components is common in life. Cold air flows past the surfaces of electronic components and carries heat away. Its characteristic is that there is always a thin layer of fluid near the wall surface, in which heat dissipation depends on heat conduction. However, outside the thin layer of fluid, heat dissipation mainly depends on convection. The formula for convective heat exchange always follows Newton's law of cooling. The formulas are as below:

$$
\begin{aligned}
& Q_{\alpha}=h_{c} A\left(T_{w}-T_{f}\right), \\
& q_{\alpha}=h_{c}\left(T_{w}-T_{f}\right) .
\end{aligned}
$$

There are some assumptions that need to be met before using this model [18]:

1. The air flow is incompressible.

2. Flow field is turbulent. 
3. Fluid density changes only affect buoyancy.

4. The data center walls are all the same materials, and the thermal conductivity remains stable.

5. Air density is constant, kinematic viscosity coefficient is constant, specific heat capacity is constant, and thermal conductivity is constant.

6. Do not consider the influence of humidity.

\subsection{Boundary conditions}

Boundary conditions for simulation are listed in Table 2. The room wall temperature is constant 20 degrees and thermal conductivity is $1.7(w / m \cdot k)$. Wall material of racks is steel, and fans at the back of racks are simulated using a simplified fan model with a pressure jump of 20Pa. The pressure jump is determined by the mass of air flow. In this case, the air flow rate of each rack is $300 \mathrm{~m}^{3} / \mathrm{h}$. A total of 30 fans with size of $180 \mathrm{~mm} \times 180 \mathrm{~mm} \times 60 \mathrm{~mm}$ and round speed of $2700(R / \mathrm{min})$ are required, so the mass of air flow for each fan is $10 \mathrm{~m}^{3} / \mathrm{h}$, which is approximately 6CFM. By consulting the fan performance curve, the static pressure is about $20 \mathrm{~Pa}$ when the mass of air flow is $6 \mathrm{cfm}$. The pressure jump is a constant in this paper, if it is a polynomial, we can use the formula below. Servers inside cabinets are rectangular and its surface material is aluminum. Each sever generates heat at a rate of 500w. Convective heat transfer coefficient on the server surface is set to $7.6\left(w / m^{2} \cdot k\right)$ according to the formula (3.3b) and (3.3c). Conditioners wall material is also steel. The inlet speed is $3 \mathrm{~m} / \mathrm{s}$ and the outlet is set to be pressure

Table 2: Boundary conditions.

\begin{tabular}{||l|l||}
\hline Boundary & Boundary conditions \\
Room wall & Constant temperature of $20^{\circ} \mathrm{C}$ \\
Heat & conductivity of $1.7(w / m \cdot k)$ \\
Cocks wall & Steel \\
Cold aisle top wall & Steel \\
Sever surface & Aluabatic \\
Convective & heat transfer coefficient of $7.6\left(\mathrm{w} / \mathrm{m}^{2} \cdot \mathrm{k}\right)$ \\
Heat & generation rate of $500 \mathrm{w}$ \\
Inlet & Velocity with $3 \mathrm{~m} / \mathrm{s}$ \\
Constant & temperature of $22^{\circ} \mathrm{C}$ \\
Outlet & Pressure outlet of $0 \mathrm{~Pa}$ \\
Fan & Pressure jump of $20 \mathrm{~Pa}$ \\
\hline
\end{tabular}


outlet. At the top of cold aisle is adiabatic

$$
\begin{aligned}
& \Delta \mathrm{p}=\sum_{n=1}^{N} f_{n} v^{n-1}, \\
& N u=0.664 \operatorname{Re}^{1 / 2} \operatorname{Pr}^{1 / 3}, \\
& h_{c}=\frac{\lambda}{l} N u .
\end{aligned}
$$

\subsection{Mesh sensitivity verification}

Each step in the CFD simulation process needs to be as efficient and reliable as possible. Mesh generation is one of the most time-consuming step, especially for three dimensional models [19]. Structured and unstructured meshes can be generated using commercial software. In the three dimensional model, generating structured mesh is difficult and time-wasted. Therefore, unstructured mesh is selected and generated with software. Hexahedral meshes are easier generated and can capture geometric features; furthermore they are also better quality for geometric models which are all rectangular. In addition, symmetric methods can be used on this symmetric model to reduce half of the mesh quantity. Three different numbers of grids has been provided in the calculations, which are 6 million, 7 million and 9.5million, respectively. Numerical simulation results are plotted in Fig. 2 and Fig. 3.

As results depicted above, temperature at different heights in location $\mathrm{A}$ is almost the same for three different meshes. In location $B$, the difference is also very small. The maximum temperature difference between them is no more than $0.5^{\circ} \mathrm{C}$. This proves that 6 million grids are accurate enough for the present simulation and the error is in an acceptable range. In this way, less time will be consumed on simulation and lower hardware requirements need to be met [20].

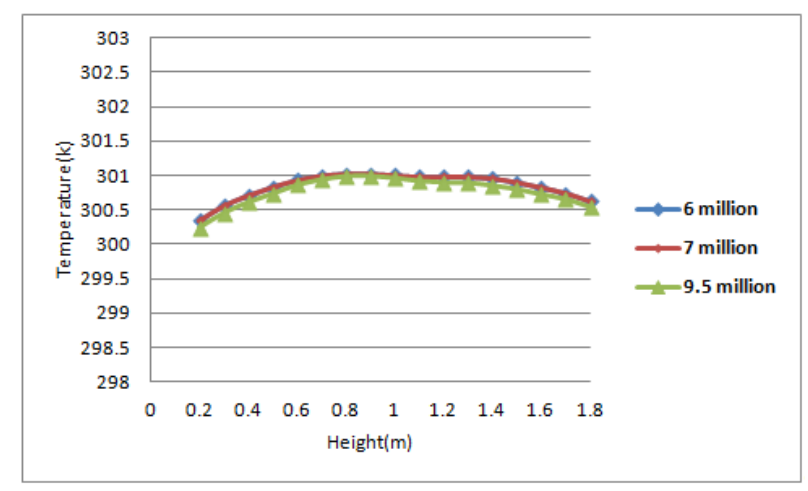

Figure 2: Temperature comparison for different grids results in location A. 


\subsection{Validation of CFD method}

In CFD simulation, a necessary step is to compare simulation results with the experimental results [21]. By comparing results, the method used to simulation can be proved correct or not. If the error is within a reasonable range, then we can take the next step. In Fig. 4, maximum air temperature at $0.2 \mathrm{~m}$ height, $1.0 \mathrm{~m}$ height, $1.8 \mathrm{~m}$ height, inlet and outlet of racks and in the whole room is compared with the results of Liu. This geometry model is not exactly the same as experimental one. There are some differences between them. The grid used for the calculation may also be of poor quality in some places, leading to a rise in the maximum temperature. Though simulation temperature is slightly higher than Liu's, the overall trend is same and the error is acceptable. It is low in the bottom of racks, then rise with the increase of height. The maximum air temperature is in outlet of racks, which is also the maximum air temperature in the whole room and represents the conditioners operating environment.

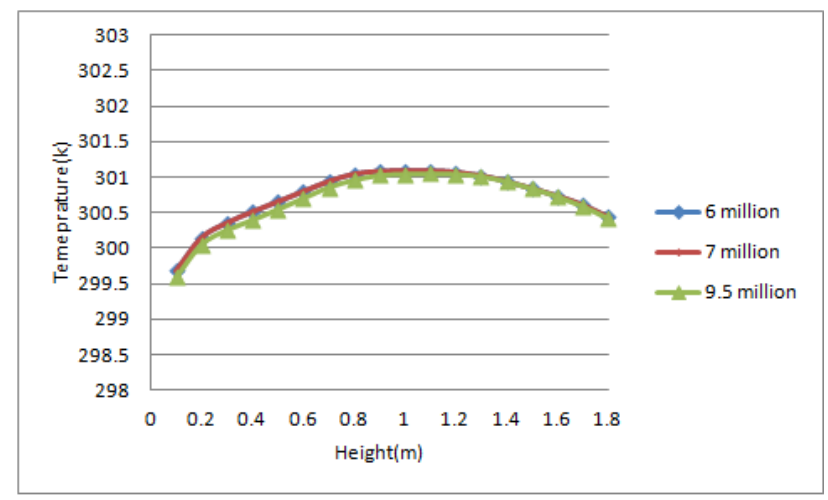

Figure 3: Temperature comparison for different grids results in location B.

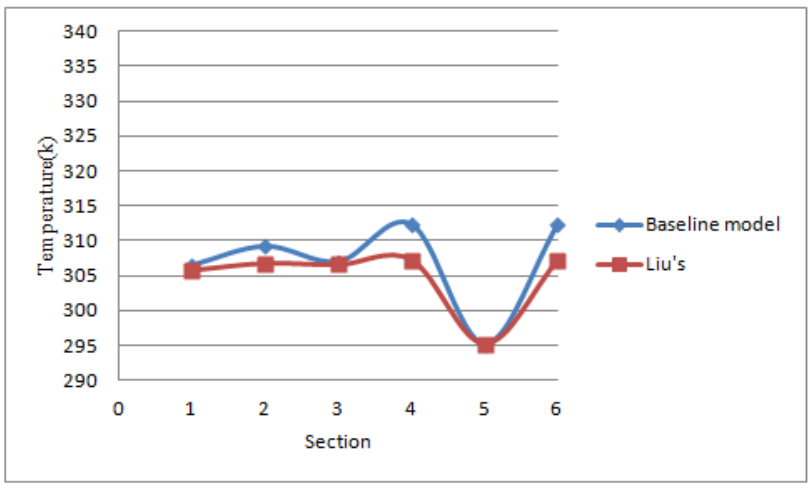

Figure 4: Temperature comparison in different sections. 


\section{Optimization of air distribution}

The air flow distribution will directly affect heat dissipation. One of the advantages of CFD simulation is the instant visualization of the air flow and the temperature field. Air flow at hot point is usually blocked and velocity is lower than other places. Besides reducing hot points, another goal is to make temperature of severs lower and more even. Temperature uniformity can affect the stability and service life of servers.

\subsection{Baseline model}

As displayed in Fig. 2, temperature rises to the maximum at $1.5 \mathrm{~m}$ height. A slice of temperature contours is made at the same height which is shown in Fig. 5(a). It is obviously seen that temperature is higher for two racks which are marked with arrows in Fig. 5(b). The velocity for surfaces of these two racks is also slower than other fans. The face-to-face arrangement results in uneven air distribution and hot points. Fig. 6 reveals that there are vortices at hot points. The streamlines show that cold air from the inlet flows along the cold aisle, and then turns around when it collides with another air-stream or a wall. At the two racks which are marked using the arrow, the cold air is obstructed by walls of racks. It is clearly seen that vortices appear near the wall and heat can not be dissipated.

\subsection{Rearrangement of air conditioners and racks}

In this paper, the location of conditioners has been changed to a diagonal arrangement. There are two things needing to be noted: Firstly, the cold air is rough to reach racks at the corner of room. It is reasonable to place the air conditioner at the corner to prevent airflow uneven. Secondly, making air conditioners distribute as evenly as possible. However, in the wall of racks, the airflow is still blocked. Since the cold air supplied from the inlet of air conditioners is not angled in the velocity, it is just facing the cabinet. The temperature of the racks next to racks facing the air conditioner is higher. By opening holes in side

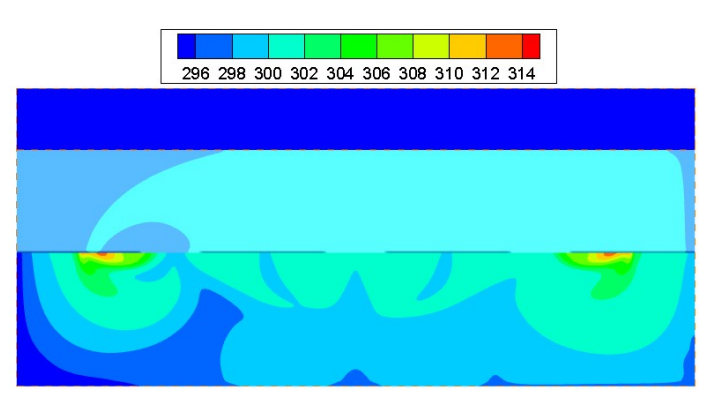

(a)

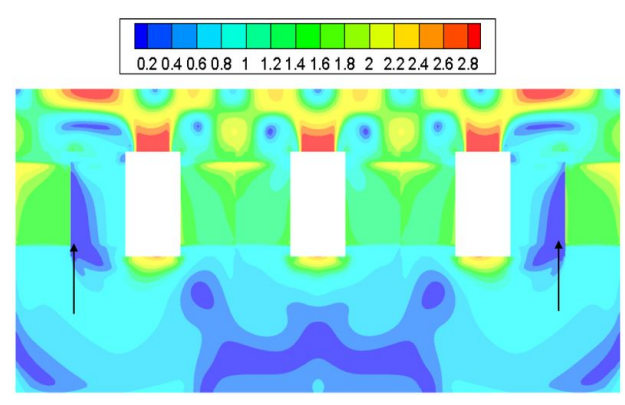

(b)

Figure 5: Temperature and velocity contours at $1.5 \mathrm{~m}$ height for baseline model. 


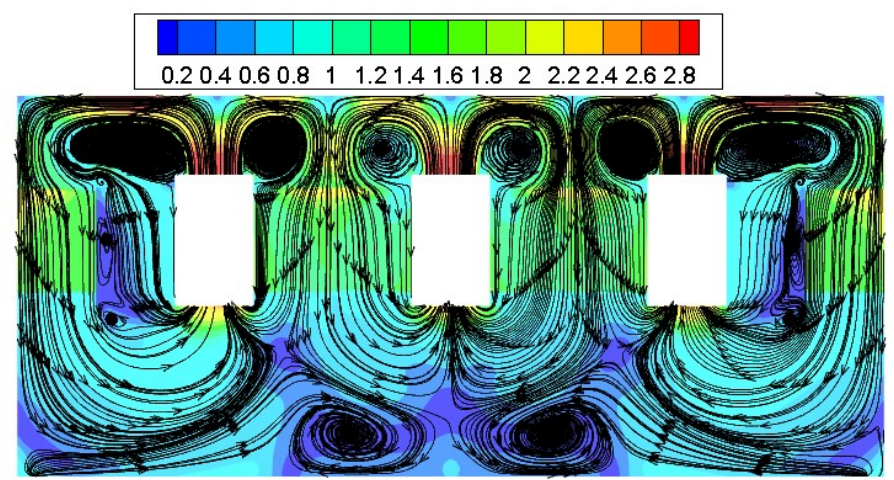

Figure 6: Streamline at $1.5 \mathrm{~m}$ height for baseline model.

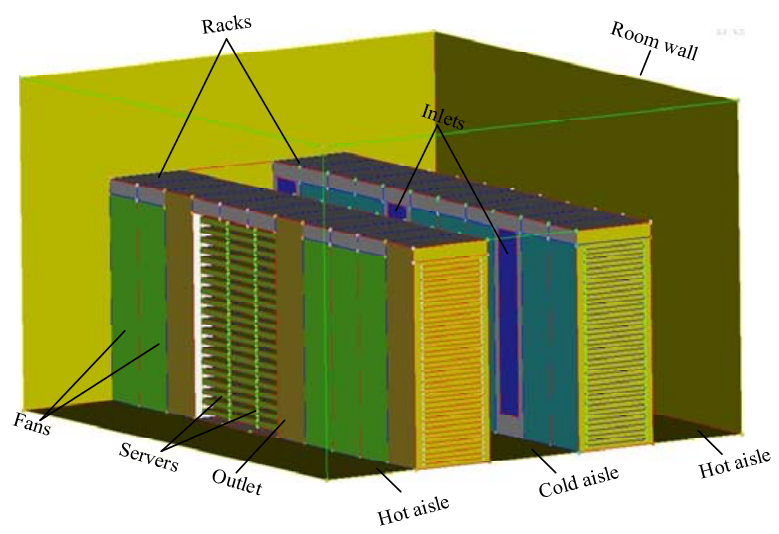

Figure 7: Rearrangement model.

walls of racks, cold air can pass through the wall to reach racks on both sides.

Compared with the baseline model, the rearrangement of conditioners and racks is shown in Fig. 7. In order to reduce the interference of airflows, the position of the air conditioner is staggered. To ensure cold air reach the corner, one conditioner needs to be placed at the end of each row. The other conditioners are arranged in a uniform distance.

Results from Fig. 8(a) show that there still exist hot points, but the maximal temperature has reduced to about $304 \mathrm{~K}$. At the back of racks, the temperature is more even than the baseline model. Fig. 8(b) plots the velocity cloud chart for new model. As shown in the figure, the velocity of cold air flowing through severs is faster and more uniform. The streamline chart from Fig. 9 shows that vortex near the wall of racks has reduced and eddy intensity is also significantly weakened.

Fig. 10 and Fig. 11 plot temperature comparison of racks back in the middle point between new and baseline model at $1.5 \mathrm{~m}$ and $1 \mathrm{~m}$ height, respectively. As shown in the line chart, the baseline model has a large temperature change at heights of $1.5 \mathrm{~m}$ and $1 \mathrm{~m}$, es- 


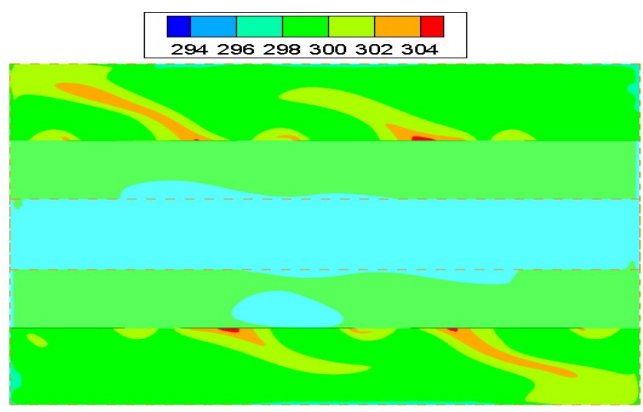

(a)

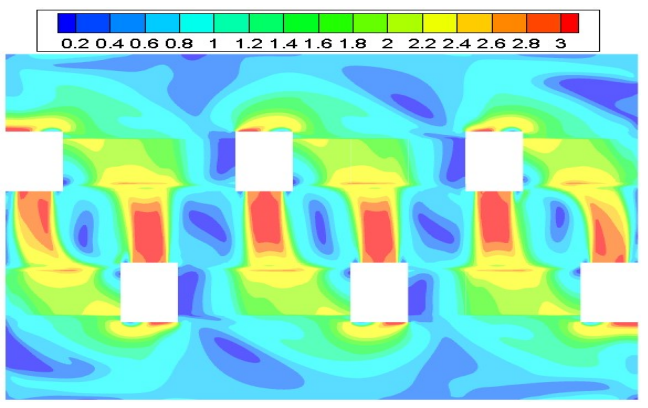

(b)

Figure 8: Contours of temperature and velocity at $1.5 \mathrm{~m}$ height for new geometric model.

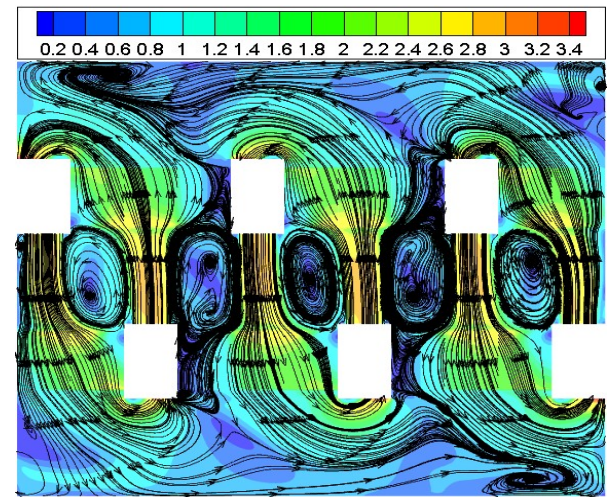

Figure 9: Streamline at $1.5 \mathrm{~m}$ height for new geometric model.

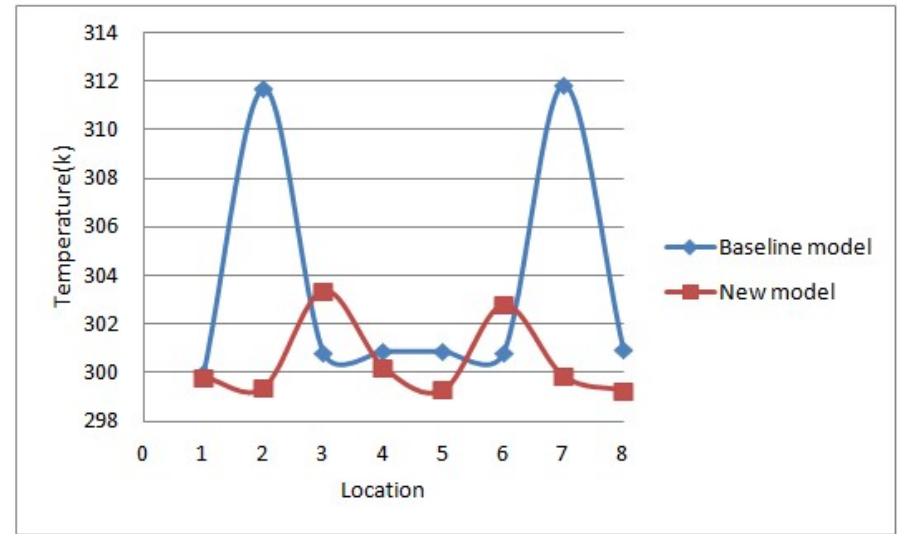

Figure 10: Temperature comparison at $1.5 \mathrm{~m}$ height.

pecially near the vortex area which is close to conditioners. For new model, temperature for hot points decreases and it changes smoothly at both two heights. 


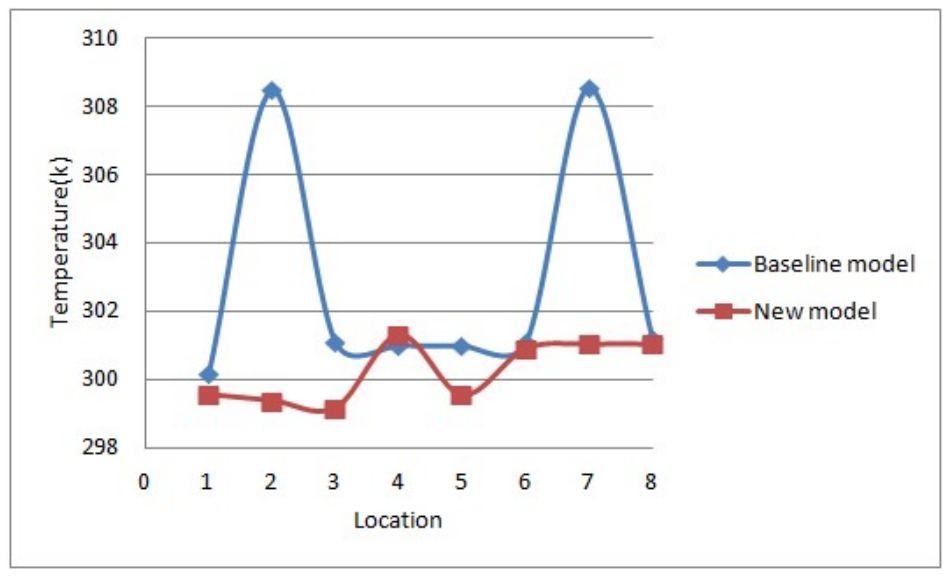

Figure 11: Temperature comparison at $1 \mathrm{~m}$ height.

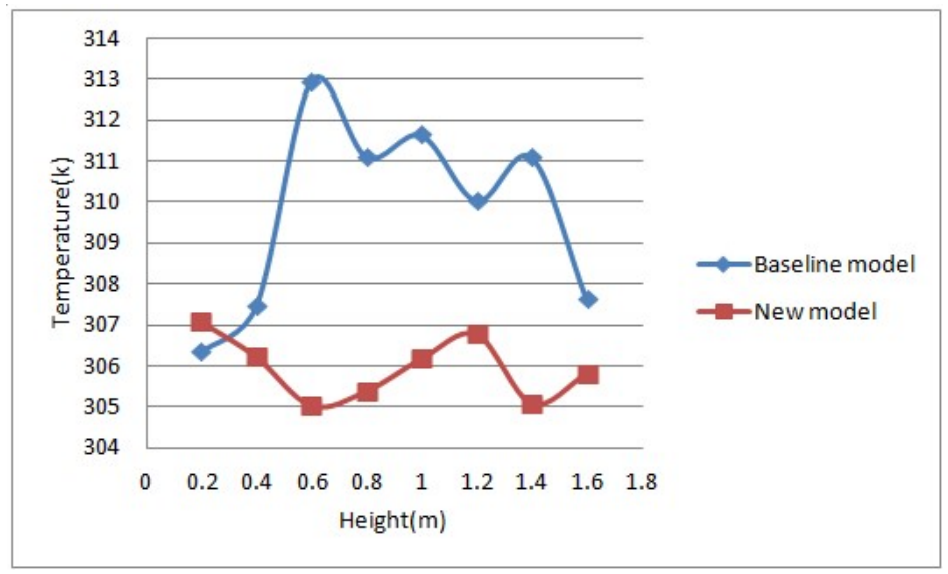

Figure 12: Comparison of maximal temperature at different heights.

In Fig. 12, the maximal temperature is compared in different heights between new and baseline model. As shown in the chart, the maximal temperature changes about $2{ }^{\circ} \mathrm{C}$ for new model. Severs will operate more stable for smaller temperature change. For baseline model, the temperature is low at the first and second racks, and then it rises rapidly at next rack. This will lead to the instability in operation and decrease of service life.

The maximum temperature on sever surface of baseline model is $71.04^{\circ} \mathrm{C}$, while it is $69.89^{\circ} \mathrm{C}$ of new model. Fig. 13 is the maximum temperature of sever surface at $2.0 \mathrm{~m}$ height. For new model, the temperature changes smoother than baseline model. The maximum temperature of baseline model is about ten degrees higher than the maximum temperature of new model. 


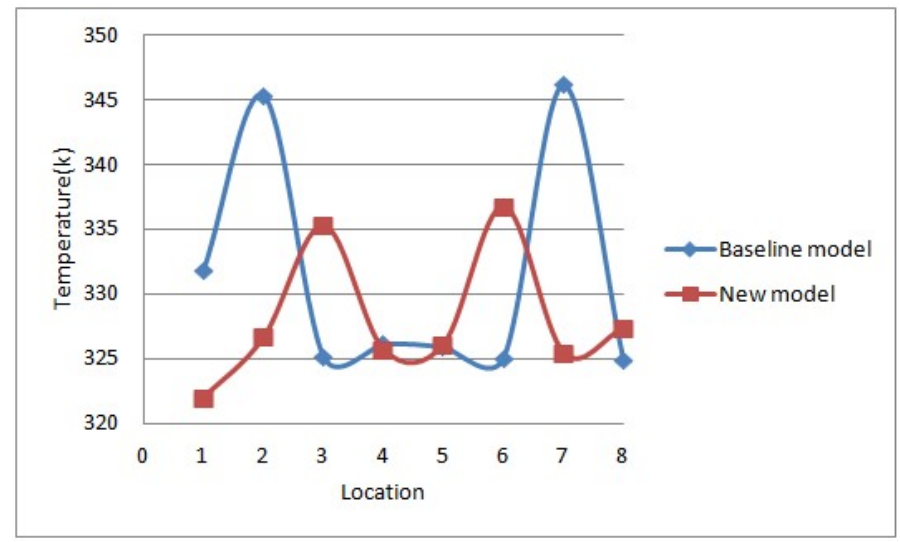

Figure 13: Temperature comparison of sever surface at $2.0 \mathrm{~m}$ height.

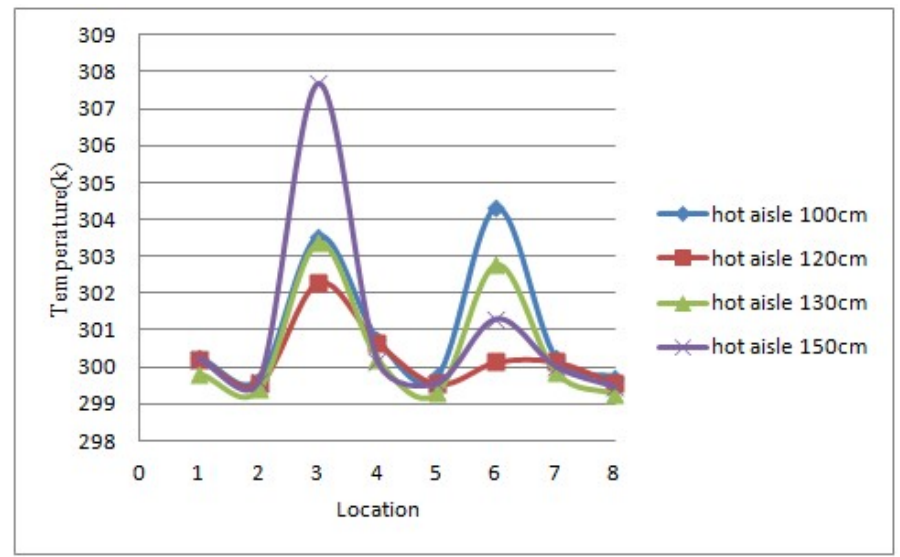

Figure 14: Temperature comparison at $1.5 \mathrm{~m}$ height for the different hot aisle distance.

\subsection{Adjustment of hot aisle distance}

Enclosed hot/cold aisle can effectively reduce the server temperature. In Lyu's paper [22], free open cold aisle, semi-enclosed cold aisle, full enclosed cold aisle and full enclosed hot aisle are studied. It proves that temperature of full enclosed hot aisle is lower than that of full enclosed cold aisle. However it requires more aisle partitions and higher cost. For this reason, only distance of hot aisle is changed in this paper. Temperature of the different hot aisle distance is simulated. Moreover, the distance of the hot aisle also affects the room area and construction cost of data center.

As shown in Fig. 14, when the distance of hot aisle is $120 \mathrm{~cm}$, temperature is lower and smoother than other ones. It displays that if the distance is too far or close, it will cause temperature to rise at $1.5 \mathrm{~m}$ height. At $1 \mathrm{~m}$ height, the change of distance results in the slight increase or decrease of temperature for $120 \mathrm{~cm}$ and $100 \mathrm{~cm}$ cases, respectively. 


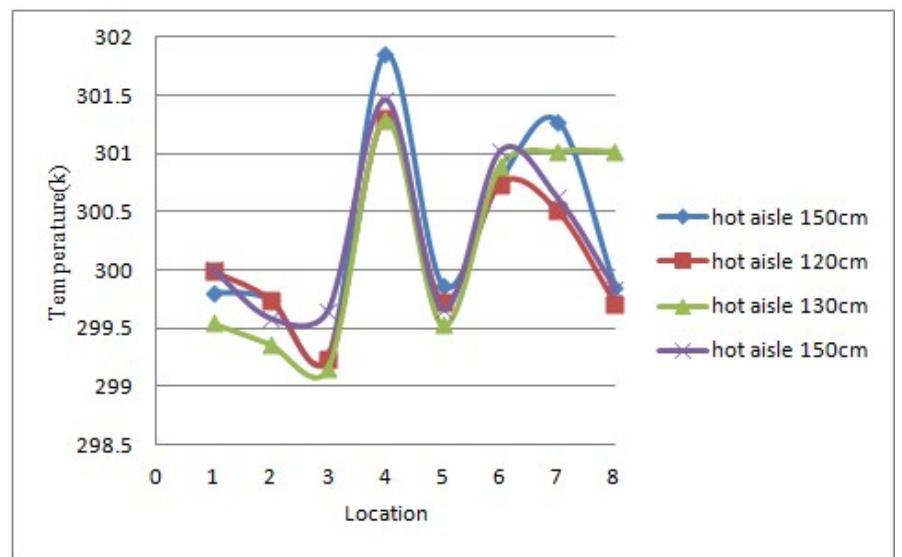

Figure 15: Temperature comparison at $1 \mathrm{~m}$ height for the different hot aisle distance.

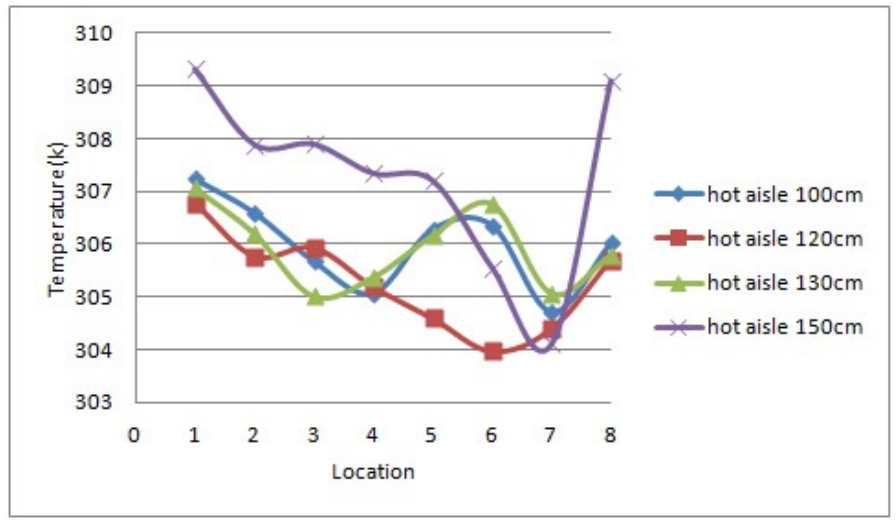

Figure 16: Maximum temperature comparison at the different height.

However, for $150 \mathrm{~cm}$ model, temperature grows obviously in location 3 . Fig. 16 reveals the maximum temperature at the different height, it is clearly seen that temperature changes intensely for $150 \mathrm{~cm}$ case. For $100 \mathrm{~cm}, 120 \mathrm{~cm}$ and $130 \mathrm{~cm}$ cases, the trend and value of temperature are slight different from location 1 to location 4 . Nevertheless, from location 4 to location 7 , the temperature is lower for $120 \mathrm{~cm}$ model. In summary, $120 \mathrm{~cm}$ model is more suitable for data center.

\section{Conclusions}

An advanced CFD simulation method was used to study the optimization of air distribution in modular data centers. By analyzing the simulation results, geometric model can be adjusted, and some conclusions were obtained in the paper, which include: 
1. Face-to-face arrangement of containers will lead to higher temperature of racks which is adjacent to the air conditioner. In this condition, temperature distribution at the back of racks is uneven.

2. Diagonal arrangement of containers can make the temperature more uniform and reduce the number of hot points and the maximum temperature. Fewer vortexes emerge near the wall than face-to-face arrangement, which indicates more efficient heat convection.

3. Opening holes in the side wall of racks facilitates the flow of cold air to reach each server. Negative wall effects which would hinder the cold air generate undesirable vortexes can be effectively reduced.

4. The distance of hot aisle influences the global distribution and the local value of temperature. In this paper, it proves that the temperature is more uniformly distribution and lower in magnitude in the case with the hot aisle of $120 \mathrm{~cm}$.

\section{Nomenclature}

CFD computational fluid dynamics

SIMPLE semi-implicit Method for pressure linked equation

$V_{\text {in }} \quad$ inlet velocity

Re Reynolds numbers

$G_{b} \quad$ generation of turbulence kinetic energy due to buoyancy

$G_{k} \quad$ generation of turbulence kinetic energy due to mean velocity gradient

g gravity

$k \quad$ turbulence kinetic energy

$\varepsilon \quad$ rate of dissipation

$h_{c} \quad$ convective heat transfer coefficient

$p \quad$ static pressure

$\rho \quad$ fluid density

$S_{h} \quad$ total volume force

$Y_{m} \quad$ contribution of the fluctuating dilatation

$S \quad$ source term

$\mathrm{Nu} \quad$ Nusselt number

$\mathrm{Pr} \quad$ Prandtl number

$\lambda$ thermal conductivity

$l \quad$ feature length 


$\begin{array}{ll}T_{f} & \text { temperature of fluid } \\ T_{w} & \text { temperature of wall } \\ A & \text { area } \\ Q_{\alpha} & \text { convective heat transfer per unit time } \\ q_{\alpha} & \text { convective heat transfer per unit area per unit time } \\ f_{n} & \text { pressure jump polynomial coefficients } \\ v & \text { the magnitude of the local fluid velocity normal to the fan } \\ N & \text { the number of polynomial coefficients }\end{array}$

\section{Acknowledgements}

The authors would like to thank China Scholarship Council (CSC), Aeronautics Science Foundation (No. 20163252037), and Fundamental Research Funds for the Central Universities (No. NP2017202) for their support.

\section{References}

[1] JiACHENG Ni AND XUELIAN BAI, A review of air conditioning energy performance in data centers, Renewable Sustainable Energy Reviews, 67 (2017), pp. 625-640.

[2] YuANMing Feng AND Pei XU, Energy consumption and energy Saving of communication room air conditioning, Telecommun. Tech., 2 (2009), pp. 78-80.

[3] R. I. AKhmad Mukaffi, Rizky S. Arief AND Wisnu Hendradji et Al., Optimization of cooling system for data center case study: PAU ITB data center, Procedia Engineering, 170 (2017), pp. 552-557.

[4] S. W. HAM, J. S. PARK AND J. W. JEONG, Optimum supply air temperature ranges of various air-side economizers in a modular data center, Appl. Thermal Eng., 77(77) (2015), pp. 163-179.

[5] R. LuCCheSE, J. OlsSON AND A. L. LJUNG ET Al., Energy savings in data centers: A framework for modelling and control of servers' cooling, IFAC-Papers OnLine, (2017), pp. 9050-9057.

[6] HongXi YAng, Jie Zhang AND Wei ZHAO, Calculation method of convective heat transfer coefficient in air cooled radiator simulation, Journal of Hubei University of Technology, 1 (2018), pp. 18-20.

[7] Shiming Yang and Wenquan TaO, Heat Transfer Theory (third edition), Higher Education Press, 1998.

[8] J. CHO AND B. KIM, Evaluation of air management system's thermal performance for superior cooling efficiency in highdensity data centers, Energy Build., 43 (2011), pp. 2145-2155.

[9] K. A. VAIBHAV, S. ViKNESHAN AND J. YOGENDRA, Thermal characteristics of open and contained data center cold aisle, J. Heat Transfer, 135 (2013), 061901.

[10] S. A. NADA, K. A. ElFEKY AND A. M. A. ATTIA, Experimental investigations of air conditioning solutions in high power density data centers using a scaled physical model, Int. J. Refrigeration, 63 (2016), pp. 87-99.

[11] J. PRIYADUMKOL AND C. KitTICHAIKARN, Application of the combined air-conditioning systems for energy conservation in data center, Energy Buildings, 68(1) (2014), pp. 580-586. 
[12] J. CHO, J. YANG AND W. PARK, Evaluation of air distribution system's airflow performance for cooling energy savings in high-density data centers, Energy Buildings, 68 (2014), pp. 270-279.

[13] D. WiLsON, Cooling system design for data centers utilizing containment, Archit Ashrae Trans., 118 (2012), pp. 415.

[14] XiaOlei YUAN, JinXiang LiU And Yujiang YANG ET AL., Investigation and improvement of air distribution system's airflow performance in data centers, Procedia Eng., 205 (2017), pp. 2895-2902.

[15] FANG LIU, ZHIGANG WANG AND QUNLI ZHANG, The application of a modular air conditioning system in data center, Procedia Eng., 205 (2017), pp. 2600-2606.

[16] J. D. Posner, C. R. Buchanan And D. Dunn-Rankin, Measurement and prediction of indoor air flow in a model room, Energy Build., 35(5) (2003), pp. 515526.

[17] RuOXUN WU, Study on energy-saving design of storage high-heat-density computer room air conditioning system, Harbin Institute of Technology, 2012.

[18] Afshin Amani, Elham Jalilnejad And Seyyed Mohammad Mousavi, Simulation of phenol biodegradation by Ralstonia eutropha in a packed-bed bioreactor with batch recycle mode using CFD technique, J. Industrial Eng. Chem., 59 (2018), pp. 310-319.

[19] ZAib Ali, PAUl G TUCKer AND SHAhroKH ShAHPAR, Optimal mesh topology generation for CFD, Comput. Methods Appl. Mech. Eng., (2016), pp. 317.

[20] MURIEL LTEN, SHULi LiU AND ASHISH SHUKLA, Experimental validation of an air-PCM storage unit comparing the Effective Heat Capacity and Enthalpy methods through CFD simulations, Energy, (2018).

[21] SMITH BARTON L, The difference between traditional experiments and CFD validation benchmark experiments, Nuclear Eng. Design, 312 (2017), pp. 42-47.

[22] Chao Lyu, Guangming Chen And ShuiQuan Ye ET Al., Enclosed aisle effect on cooling efficiency in small scale data center, Procedia Eng., 205 (2017), pp. 3789-3796. 